\title{
Molecular basis of Antigenic Reassortment of Influenza Virus
}

\author{
Mohammed Helmy Faris Shalayel* \\ Professor of Biochemistry, University of Hafr Al-Batin, Saudi Arabia
}

*Corresponding author: Mohammed Helmy Faris Shalayel, Department of Pharmacology Practice, University of Hafr Al-Batin, Saudi Arabia.

To Cite This Article: Mohammed Helmy Faris Shalayel, Molecular basis of Antigenic Reassortment of Influenza Virus. 2020 - 8(1). AJBSR. MS.ID.001223. DOI: 10.34297/AJBSR.2020.08.001223.

Received: 濫 February 11, 2020; Published: 眥 March 09, 2020

\begin{abstract}
Frequent pandemic alerts were raised by the World Health Organization in response to newly emerging influenza strains. This minireview highlights the dynamic alterations in the viral adaptability resulted from antigenic drift and reassortment that enable flu virus to escape recognition by the host immune system and neutralization by antibodies produced in preceding infections or vaccinations.
\end{abstract}

Keywords: Influenza, Antigenic drift, Antigenic reassortment, Vaccine

\section{Introduction}

Influenza is a serious disease that causes outrageous morbidity and mortality in every year with its great impact on either extreme of the age range [1]. Influenza viruses are classified into three categories A, B, and C. Of which, type B can exclusively infect only humans. Type $\mathrm{C}$ infects human and swine while type $\mathrm{A}$ affects multiple host organisms including humans, marine mammals, equine, swine, and avian [2].

In 1997, H5N1, a highly pathogenic avian influenza virus, gave rise to its first human infection in Hong Kong [3] and the fatality rate reached 53\% in reports of 2003 [4]. In April 2009, a high pandemic alert was raised by the World Health Organization after a new swine-origin influenza (H1N1) emerged in Mexico and the United States that quickly spread via human-to-human transmission to 30 countries and more or less 208 countries reported this swine flu by December of the same year $[5,6]$.

According to the serological reactivity of their surface glycoproteins, designated as hemagglutinin (HA) and neuraminidase (NA), influenza A viruses are classified into subtypes. Sixteen HA (H1 to H16) and 9 NA (N1 to N9) subtypes have been identified in wild aquatic birds in the last century. Detection of this virus in new species highlights a raised prospect for cross-species transmission [7].

\section{Viral Surface Proteins}

The HA protein is synthesized as a single-chain precursor (HA0) during viral replication and is subsequently cleaved by host proteases into the functional/infectious HA1/HA2 form [8].

If there are small mutations in the genes of influenza viruses that can lead to alterations in the surface proteins of the virus, this called antigenic drift [9]. Hence, hemagglutinin and neuraminidase are two surface proteins of the influenza A virus that play fundamental intermediary roles in initiating pathogenicity during virus entry into host cells and release from infected cells [10]. Influenza A viruses attach to cells through HA binding to terminal sialic acids of glycoproteins on the surfaces of respiratory epithelial cells. The host range of infectivity is defined mainly by influenza A virus affinity for different sialosides. Avian viruses like H5N1 and H7N9 bind to sialic acid linked to galactose via an $\alpha 2-3$ bond, and human viruses discriminatorily bind to sialate attached to galactose through an $\alpha 2-6$ bond [11].

Using super-resolution microscopy and site-specific fluorescent labelling, Vahey and Fletcher (2019) revealed that HA and NA are asymmetrically distributed on the surface of filamentous viruses, producing a spatial organization of binding and cleaving activities that enables viruses to stride continuously away from their NA-rich 
swivel. This organized architecture helps virus to penetrate host mucus barrier [12].

Dynamic alterations in the viral adaptability resulted from antigenic drift and functional mutations in the surface proteins may lead to possible emergence in production of more virulent strains [13]. Owing to the interlinkage between these two surface proteins, some HA mutations were found to affect the adaptive landscape of NA, However, the amplitude to which emergence of one protein affects that of the other one is under research. Hence, the adaptive landscape of a viral protein is notably sensitive in terms of the entire genomic context [14].

In spite of being in constant emergence and evolution, these two glycoproteins HA/NA balance is likely to persist equiponderant in human viruses as an optimal balance is needed to preserve optimal viral competence. Hence, HA/NA balance and interplay between the host receptor and influenza virus surface proteins are quite important to explore evolution and emergence of influenza viruses $[15,16]$.

\section{Antigenic Reassortment}

Formation and release of newly formed virion is initiated by the action of NA that hydrolyzes terminal sialic acid moiety from cell receptors thus leads to avoiding immobilization by sialic acid in host mucus, prevention of the aggregation of virus particles and spread of infection $[12,17]$. Antigenic reassortment or shift occurs when strain of two or more different viruses, merge to produce a novel subtype having a combination of the surface antigens of the two or more parental strains [18,19]. Hence, reassortment is a time-dependent natural mutation of recognized strains of influenza which may lead to failure of immunological recognition and vaccine mismatch [18]. For human seasonal influenza virus. A (H3N2) Has, five antigenic sites (A, B, C, D, and E) have been previously characterized [11]. The molecular basis of the virus to escape recognition by the immune system is that the virus' replication machinery lacks a proofreading mechanism consequently mutations in flu virus, as in all RNA viruses, are happening in frequent manner. This accounts for vaccine mismatch and annual change of vaccine composition due to the different annual circulating strains [20]. Reassortment is the most complicated form of viral antigenic shift. It happens when the same animal is simultaneously infected by two viruses. An example is when pigs are infected by both human and avian influenza strains. Hence, A hybrid virus is created from reassortment of genetic materials from both viruses within the infected porcine cells. In the last 115 years, more or less seven antigenic reassortments in type A influenza have been attested [21]. Thus, antigenic reassortment and variations of influenza $A$ viruses are evolved by genomic mutations that occur in the transmembrane protein HA1, triggering the viral capability to avoid neutralization by antibodies produced in preceding infections or vaccinations. Hence, prognostication of these antigenic events is useful to understand viral epidemiology and to expand insights into the evolutionary mechanisms reinforcing viral antigenic divergence as well as to design new influenza vaccines [22].

Gouma et al. [23] reported that the egg- adapted H3N2 component of the 2019 Southern Hemisphere influenza vaccine educed an antibody response in ferrets that is substantially triggering hemagglutinin antigenic site A. This finding represents a dilemma as antigenic site A of hemagglutinin substitutions are prevalent in most of the currently circulating H3N2 viruses in the Southern Hemisphere [23]. Although the delineation of the global influenza vaccine evolution targeting the HA viral domain, there are still some knowledge incompetence concerning immunological hallmarks. Studies of sequential influenza virus infection in animal models may be utilized to improve knowledge beyond how the human immune response modulates over sequential influenza infections and vaccinations and ameliorate vaccination strategies as well as our ability to produce more efficient vaccines [24].

\section{Conclusion}

Dynamic alterations in the viral adaptability due to frequent antigenic drift and functional mutations in the surface proteins may lead to possible emergence in formation of highly virulent strains. The molecular basis of the virus to escape recognition by the immune system is that the influenza virus' replication machinery lacks a proofreading mechanism consequently, mutations are frequent in flu virus. Antigenic reassortment occur when strain of two or more different viruses, merge to develop a novel subtype having hybrid surface antigens of many different strains. Knowledge incompetence concerning immunological hallmarks beyond frequent antigenic shift of the virus is constituting a dilemma in terms of the variabilities and efficacy of vaccines against flu virus.

\section{References}

1. Treanor J (2004) Influenza vaccine-out maneuvering antigenic shift and drift. N Engl J Med 350(3): 218-220.

2. Mandell GL, Bennett JE, Dolin R (2010) Mandell, Douglas, and Bennett's principles and practice of infectious diseases. Influenza viruses including avian influenza and swine influenza 2: 2265-2288.

3. Yuen KY, Chan PK, Peiris M, Tsang DN, Que TL, et al. (1998) Clinical features and rapid viral diagnosis of human disease associated with avian influenza A H5N1 virus. Lancet 351(9101): 467-471.

4. (2015) Cumulative number of confirmed human cases for avian influenza A(H5N1) reported to WHO. World Health Organization, Switzerland.

5. Smith GJD, Vijaykrishna D, Bahl J, Lycett SJ, Worobey M, et al. (2009) Origins and evolutionary genomics of the 2009 swine-origin H1N1 influenza A epidemic. Nature 459(7250): 1122-1125.

6. AlMazroa MA, Memish ZA, AlWadey AM (2010) Pandemic influenza A (H1N1) in Saudi Arabia: description of the first one hundred cases. Ann Saudi Med 30(1): 11-14.

7. Yang H, Nguyen HT, Carney PJ, Guo Z, Chang JC, et al. (2015) Structural and functional analysis of surface proteins from an A(H3N8) influenza virus isolated from New England harbor seals. J Virol 89(5): 2801-2812. 
8. Hulse-Post DJ, Sturm-Ramirez KM, Humberd J, Seiler P, Govorkova EA et al. (2005) Role of domestic ducks in the propagation and biological evolution of highly pathogenic H5N1 influenza viruses in Asia. Proc Natl Acad Sci U S A 102(30): 10682-10687.

9. (2019) Centers for Disease Control and Prevention, National Centre for Immunization and Respiratory Diseases (NCIRD). Influenza (flu).

10. Webster RG, Bean WJ, Gorman OT, Chambers TM, Kawaoka Y (1992) Evolution and ecology of influenza A viruses. Microbiol Rev 56(1): 152 179.

11. Hua Yang, Paul J Carney, Vasiliy P Mishin, Zhu Guo, Jessie C Chang, et al. (2016) Molecular Characterizations of Surface Proteins Hemagglutinin and Neuraminidase from Recent H5Nx Avian Influenza Viruses. Journal of Virology 90(12): 5770-5784.

12. Vahey MD, Fletcher DA (2019) Influenza A virus surface proteins are organized to help penetrate host mucus. Elife 8: e43764.

13. Jones S, Nelson-Sathi, Wang, Y, Raji Prasad, Sabrina Rayen, et al. (2019) Evolutionary, genetic, structural characterization and its functional implications for the influenza A (H1N1) infection outbreak in India from 2009 to 2017. Sci Rep 9: 14690.

14. Neverov AD, Kryazhimskiy S, Plotkin JB, Bazykin GA (2015) Coordinated Evolution of Influenza A Surface Proteins. PLoS Genet 11(8): e1005404.

15. Gaymard A, Le Briand N, Frobert E, Lina B Escuret V (2016) Functional balance between neuraminidase and haemagglutinin in influenza viruses. Clin Microbiol Infect 22(12): 975-983.
16. Byrd-Leotis L, Cummings RD, Steinhauer DA (2017) The Interplay between the Host Receptor and Influenza Virus Hemagglutinin and Neuraminidase. Int J Mol Sci 18(7): 1541.

17. Liu C, Eichelberger MC, Compans RW, Air GM (1995) Influenza type A virus neuraminidase does not play a role in viral entry, replication, assembly or budding. J Virol 69(2): 1099-1106.

18. Zambon Maria C (2008) Epidemiology and pathogenesis of influenza. Journal of Antimicrobial Chemotherapy 44: 3-9.

19. Dawood S, Bresee J (2018) Influenza Viruses. Principles and Practice of Paediatric Infectious Diseases Elsevier 229: 1181- 1190.

20. (2018) World Health Organization. How pandemic influenza emerges.

21. Flaherty DK (2012) Immunology for pharmacy. Vaccine-Preventable Diseases pp. 197-213.

22. Degoot AM, Adabor ES, Chirove F, Wilfred Ndifon (2019) Predicting Antigenicity of Influenza A Viruses Using biophysical ideas. Sci Rep 9(10218).

23. Gouma S, Weirick M, Hensley SE (2019) Potential Antigenic Mismatch of the H3N2 Component of the 2019 Southern Hemisphere Influenza Vaccine. Clin Infect Dis 11: ciz723.

24. Francis ME, King ML, Kelvin AA (2019) Back to the Future for Influenza Preimmunity-Looking Back at Influenza Virus History to Infer the Outcome of Future Infections. Viruses 11(2): 122. 